\title{
IT-basierte Entscheidungs- unterstützung am Beispiel der Subprimekrise: Wunderwaffe oder Teufelszeug?
}

DOI 10.1007/s11576-008-0093-2

\section{Der Autor}

Prof. Dr. Hans Ulrich Buhl

Universität Augsburg Lehrstuhl für BWL, Wirtschaftsinformatik, Informations- \& Finanzmanagement Universitätsstraße 16 86135 Augsburg Deutschland hans-ulrich.buhl@ wiwi.uni-augsburg.de
In Heft 1/1994 wurde das von der WKWI beschlossene „Profil der Wirtschaftsinformatik" veröffentlicht. Gemäß diesem versteht sich die Wirtschaftsinformatik als interdisziplinäre Wissenschaft mit Informations- und Kommunikationssystemen (IKS) als zentralem Gegenstand. IKS sollen dabei im Sinne eines zentralen Nervensystems eines Unternehmens die Zahlungs- und Leistungsflüsse innerhalb betrieblicher Prozesse durch den Austausch von Informationen koordinieren sowie Entscheidungsträger als Voraussetzung für ein erfolgreiches unternehmerisches Handeln mit relevanten Informationen versorgen. Das ist erforderlich, weil in Unternehmen laufend operative und strategische Entscheidungen getroffen werden. Führt man sich zudem vor Augen, dass pro Jahr ca. 5 Exabyte $\left(=5^{*} 10^{18}\right.$ Bytes) an Informationen neu entstehen - dies entspricht ungefähr der Datenmenge aller je vom Menschen gesprochenen Worte - so erscheint der stark wachsende Einsatz von IT zur Selektion, Verarbeitung und Bereitstellung von Informationen nach wirtschaftlichen Kriterien nicht nur logisch, sondern sogar zwingend notwendig.

Natürlich ist die extrem große Menge an neu entstehenden Informationen z. T. auch auf den Einsatz von IT zurückzuführen. Jedoch bietet die IT auch die Möglichkeiten, mit dieser Informationsfülle im Sinne einer bestmöglichen Unterstützung von Entscheidungsprozessen umzugehen. Zentrales Element hierbei bilden spezialisierte Anwendungssysteme wie Entscheidungsunterstützungssysteme (EUS) als Teil von IKS.

Auf diesem Gebiet kann die Wirtschaftsinformatik bereits wertvolle Beiträge in Forschung und Praxis verzeichnen. So setzen bspw. Unternehmen vielfach Anwendungssysteme für eine teilweise bis vollständige Automatisierung von operativen Routineentscheidungen ein. Der Vorteil liegt klar auf der Hand: Mitarbeiter haben mehr Zeit, um sich kreativen, strategischen und stärker Wert schaffenden Tätigkeiten zu widmen. Aber auch auf strategischer Ebene gibt es wertvolle Errungenschaften. So helfen hier zunehmend analytische Anwendungssysteme nicht nur bei der Beschaffung, Aufbereitung und Auswahl relevanter Informationen, sondern auch bei der Generierung von Handlungsempfehlungen. Letztere beruhen in der Regel auf Entscheidungsmodellen. Beispiele hierfür finden sich u. a. im Risikomanagement, in der Logistik oder im CRM. Der sinnvolle Einsatz von EUS kann betriebliche Entscheidungsprozesse hinsichtlich Geschwindigkeit und Qualität erheblich verbessern, was zu zuverlässigeren Reaktionen auf Markt- und Umweltereignisse führt und Wettbewerbsvorteile schafft.

Das alles klingt, als ob diese Errungenschaften der Wirtschaftsinformatik als eindeutiger Fortschritt, wenn nicht gar als „Wunderwaffe“ eingestuft werden können, oder?

Der Skeptiker mag spätestens an dieser Stelle jedoch fragen, warum die teilweise heute schon exorbitant hohen Gehälter von Managern und anderen Top-Entscheidern weiter steigen. Dies wäre widersprüchlich, geht man davon aus, dass - wie eben überspitzt skizziert - EUS für einen immer größeren Anteil der getroffenen Entscheidungen „verantwortlich“ sind. Extrapoliert man diese Entwicklung, dann müssten doch streng genommen immer weniger Entscheidungen von den Führungskräften selbst getroffen und die meisten allenfalls noch „abgenickt" werden. Wenn dem so wäre, müssten wir uns berechtigterweise auch fragen, warum wir uns als Gesellschaft 
immer noch Aufsichts- und Kontrollinstanzen unter anderem für Unternehmen, deren Management und Aufsichtsorgane leisten. Warum also?

Tatsächlich sind trotz des zunehmenden EUS-Einsatzes zahlreiche unternehmerische Fehlentscheidungen teilweise erheblichen Ausmaßes beobachtbar. Das Problem liegt dabei zum einen in den implementierten Entscheidungsmodellen und zum anderen im teilweise blinden Vertrauen in diese Modelle. Prinzipiell können Entscheidungsmodelle - mögen sie noch so innovativ und Mehrwert stiftend sein - nur unter vereinfachenden Annahmen einen Teil der realen Komplexität abbilden. Ist wie oftmals der Fall - mindestens eine Annahme nicht erfüllt, so ist auch die Interpretation der Modellaussagen für eine reale Gegebenheit nicht mehr uneingeschränkt zulässig. Damit ergibt sich ein Modellrisiko, dass also Modelle die relevanten Aspekte der Realität nicht nur unvollständig, sondern fehlerhaft abbilden. Vor allem für modelltheoretische Grenzbereiche sind eine kritische Prüfung und differenzierte Bewertung der Aussagenkraft, Limitationen und Anwendbarkeit erforderlich. Zudem gilt es, Modelle kritisch auf Ihre Robustheit zu prüfen, also inwieweit sie auch bei einer Schwankung der angenommenen Parameter noch näherungsweise korrekte Aussagen liefern. Die Koinzidenz dieser immanenten Einschränkungen mit einer unreflektierten Modellgläubigkeit - die durch eine Implementierung der Modelle in EUS zusätzlich verstärkt wird - birgt die Gefahr unternehmerischer Fehlentscheidungen. Ein weiteres Problem liegt in der Verbreitung von Modellen. Nutzt bspw. ein Großteil der Marktteilnehmer dieselben Modelle, kann dies zu homogenen Erwartungen und gleichgerichtetem Handeln führen. Die Folge ist eine Verstärkung normaler Marktzyklen, die unter anderen Umständen gedämpft würden. Damit können Entscheidungsmodelle bei breiter Verwendung die Grundlage für das Entstehen und letztendlich auch für das Platzen von Marktblasen sein.

Sind damit Entscheidungsmodelle und EUS doch nicht als "Wunderwaffe", sondern als „Teufelszeug" zu verurteilen?

Vermutlich liegt die Antwort - über die sich zweifelsohne trefflich philosophieren ließe - wie immer in der Mitte. Die wirklich interessanten Fragen aus heutiger Sicht lauten jedoch:Welche gemeinsamen Anstrengungen sind nötig, um einen Schritt in Richtung einer besseren IT-gestützten Entscheidungsunterstützung zu gehen? Welchen Beitrag kann die Wirtschaftsinformatik leisten? Wie weit geht diesbezüglich die Verantwortung der Wirtschaftsinformatik angesichts der Tatsache, dass viele Entscheidungsmodelle nicht originär der Wirtschaftsinformatik entstammen?

Antworten auf diese Fragen können bspw. in der aktuellen Krise an den Immobilienkreditmärkten gefunden werden, in deren Mittelpunkt die Verbriefung von Kreditforderungen steht. Dabei werden Kreditforderungen von den vergebenden $\mathrm{Hy}$ pothekenbanken an eigens dafür zumeist von Investmentbanken gegründete Zweckgesellschaften verkauft. Die Kreditforderungen werden dort in Portfolios zusammengefasst, in unterschiedliche Risikoklassen (Tranchen) unterteilt und als vermögensbesicherte Wertpapiere - sog. Asset-Backed Securities (ABS) - an den Geldund Kapitalmärkten emittiert. Mit solchen Verbriefungstransaktionen werden also Anleihen geschaffen, die mit den Zins- und Tilgungszahlungen der unterliegenden Hypothekendarlehen bedient werden. Fallen einige der unterliegenden Kreditforderungen aus, so schlagen diese - beginnend mit der risikoreichsten Tranche in absteigender Reihenfolge - auf die Anleihen durch. Als wesentliche Informationsquelle über die Ausfallrisiken der Wertpapiertranchen dienen dabei alphanumerische Ratings, die von den Ratingagenturen mittels finanzmathematischer Modelle vergeben werden.

Mögen solche strukturierten Finanzprodukte wie ABS und die damit verbundenen Verbriefungstransaktionen noch als innovative und vorteilhafte Finanzkonstrukte gelten - schließlich ermöglichte erst diese Umwandlung von illiquiden Aktiva in handelbare Wertpapiere eine Verteilung von Risiken auf den internationalen Finanzmärkten - so wurden diese Finanzstrukturen in den vergangenen Jahren doch in die Perversion getrieben. Zum einen erzeugten diese Strukturen mit der Möglichkeit, Kreditforderungen gegen eine Prämie an Zweckgesellschaften für die Verbriefung zu verkaufen, Anreize für die Hypothekenbanken, zunehmend hochriskante Kredite an Kunden mit schlechter Bonität (Subprime) zu vergeben. Diesen lagen dabei im Wesentlichen Spekulationen auf weiterhin hohe bzw. steigende Immobilienpreise und weiterhin niedrige bzw. sinkende Zinsen zugrunde. Entsprechend stieg in den 
USA der Anteil an Subprime-Krediten bei hypothekenbesicherten Wertpapieren bis zum Jahr 2006 auf ca. $40 \%$ an. Zum anderen wurde die ursprüngliche Kreditverbriefung zu immer komplexeren Finanzstrukturen ausgebaut. So werden bspw. nicht nur Kreditforderungen, sondern auch vermögensbesicherte Wertpapiere selbst in beträchtlichem Umfang neu verbrieft und als sogenannte Collateralized Debt Obligations (CDOs) emittiert. Auch CDOs werden wiederum durch Zweckgesellschaften gekauft, in Portfolios zusammengefasst, erneut strukturiert und als Collateralized Debt Obligations squared $\left(\mathrm{CDOs}^{2}\right)$ ausgegeben. Dieser Verbriefungsprozess kann dabei im Prinzip beliebig oft mit immer größeren Volumina sowie immer weniger Transparenz bzgl. der letztendlich unterliegenden Kreditforderungen und deren Qualität wiederholt werden.

Insbesondere diese komplexen Finanzstrukturen basieren fast ausschließlich auf finanzmathematischen Modellen, die Vergangenheitsdaten unreflektiert extrapolieren. Meist kann bei mehrfach strukturierten Wertpapieren nicht mehr nachvollzogen werden, welche Kredite mit welchen Risiken den Anleihen letztendlich unterliegen. Einerseits werden die Ratings der $\mathrm{CDO}$ s und $\mathrm{CDO}^{2}$ unter Verwendung solcher Modelle berechnet. Andererseits beinhalten aber bspw. auch die Anwendungssysteme für das Risiko- oder Portfoliomanagement der Banken nahezu die gleichen Modelle zur Bewertung der Ausfallrisiken solcher Wertpapiere. Ein Großteil der beteiligten Banken und Investoren glaubten mit Hilfe dieser Modelle die tatsächlichen Ausfallrisiken der komplexen Finanztitel berechnen, bewerten und steuern zu können.

Mittels geschickter Strukturierung werden die meisten Verbriefungstransaktionen so gestaltet, dass ein Großteil der Wertpapiere mit den erstklassigen Ratings „AAA“ oder "AA" ausgezeichnet wird. Die Tatsache, dass bspw. der Freistaat Bayern mit seiner erstklassigen Bonität von der Ratingagentur Standard \& Poor's ebenfalls „AAA“ geratet ist, verleiht diesen Wertpapieren damit ein echtes Qualitätssiegel. Auch viele Banken und Investoren kamen im Rahmen ihres Portfolio- und Risikomanagements zu der gleichen Einschätzung. Ex post betrachtet hat sich dieses Gütesiegel jedoch als nicht gerechtfertigt erwiesen. Der Ausgangspunkt der Krise liegt im Jahr 2006, als die amerikanischen Immobilienpreise sanken, das Zinsniveau stieg und es zu einem entsprechend starken Anstieg der Kreditausfallraten im Subprime-Segment kam. Dieser Entwicklung folgten - im Vergleich zu den tatsächlichen Kreditausfallraten - teilweise unverhältnismäßig drastische Ratingherabstufungen einer Vielzahl teilweise erstklassig bewerteter Wertpapiere in den Status von Junk Bonds und damit verbundene, massive Wertverluste. So verloren bis Juli 2008 zahlreiche „AAA“geratete $A B S$ und $C D O$ s im Durchschnitt knapp $60 \%$ ihres ursprünglichen Nominalwerts. Viele Wertpapiere mit „AA“-Rating verzeichneten im selben Zeitraum sogar einen durchschnittlichen Wertverlust von über $90 \%$. Folglich erlitten zahlreiche Investoren empfindliche Verluste. Insgesamt schätzt der Internationale Währungsfonds (IWF) die weltweiten Wertberichtigungen bis August 2008 auf 950 Mrd. US\$, davon rund $500 \mathrm{Mrd}$. US\$ bei Banken. Alleine die Investmentbank Merrill Lynch, die ein CDO-Portfolio mit einem ursprünglichen Wert von gut 30 Mrd. US\$ in ihrem Besitz hielt, verzeichnete im Zeitraum zwischen Juli 2007 und Juli 2008 Abschreibungen in Höhe von 23,4 Mrd. US\$ auf dieses CDO-Portfolio. Mit einem Restwert von $6,6 \mathrm{Mrd}$. US\$ verloren diese CDOs $78 \%$ ihres ursprünglichen Nominalwerts. Laut einer Studie des Hedge-Fonds Bridgewater Associates wird ein weiteres Anschwellen der weltweiten Verluste auf insgesamt 1.600 Mrd. US\$ erwartet. Als vorläufiger Höhepunkt der Krise verloren nahezu alle Marktteilnehmer das Vertrauen in die Wertpapiere und der weltweite Handel mit vermögensbesicherten Wertpapieren brach ein. Der Markt wurde illiquide. Sogar Wertpapiere, denen hochsolide Kreditforderungen unterliegen, wurden teilweise abgewertet und zeitweise nicht mehr gehandelt. Zeitgleich wurden immer mehr Nachrichten von namhaften Banken, die im Zuge der Krise unmittelbar von der Insolvenz bedroht waren, sowie von spektakulären Rettungsaktionen bekannt. Mit Bear Stearns, Nothern Rock, Fannie Mae und Freddi Mac, SachsenLB oder der IKB seien hier nur ein paar wenige prominente Beispiele genannt. Spätestens an dieser Stelle stellt sich zwangsläufig die Frage, wie solche massiven Ratingherabstufungen mit den beschriebenen Konsequenzen überhaupt möglich waren. Schließlich steht ein „AAA“- oder "AA“-Rating für eine sehr hohe und stabile Kreditqualität. Nach den finanzmathematischen Modellen hätte das auch nie bzw. nur mit sehr kleiner Wahrscheinlichkeit geschehen dürfen. Auch die Modelle, 
die dem Portfolio- und Risikomanagement vieler Banken und Investoren zugrunde liegen, haben ex post betrachtet das tatsächliche Risiko falsch eingeschätzt. Wo liegt nun der Fehler?

Auch hier liegt das Problem vorwiegend in den zugrunde liegenden Modellen sowie dem unreflektierten Vertrauen in deren Zuverlässigkeit und Robustheit. Letztendlich unterlagen auch die finanzmathematischen Modelle dem oben beschriebenen Modellrisiko. Zahlreiche wissenschaftliche Beiträge zeigen, dass insbesondere mehrfach strukturierte Wertpapiere wie CDOs und $\mathrm{CDOs}^{2}$ spezielle Risikostrukturen und Abhängigkeiten aufweisen. Diese werden von diesen Modellen mit ihren vereinfachenden Annahmen nicht vollständig abgebildet und können die tatsächlichen Risiken der letztendlich unterliegenden Kreditforderungen unter ungünstigen Umständen sogar potenzieren. So wurde bspw. vielfach angenommen, gewisse zukünftige Marktdaten aus historischen Daten schätzen zu können. Für die o. g. neuartigen Produkte existieren jedoch nur wenige historische Daten und keine Erfahrungswerte über Marktkrisen. Solange eine Marktsituation vorherrschte, die den bisherigen Erfahrungen - nämlich steigende Immobilienpreise und sinkende Zinsen weitestgehend entsprach, konnten zuverlässige Bewertungen generiert werden. Das aus einem Anstieg des Zinsniveaus und einem Fallen der Immobilienwerte tatsächlich resultierende und um ein vielfaches höhere Risiko für $A B S, C D O s$ und $C D O s^{2}$ konnten die Modelle jedoch nicht prognostizieren. Nahezu alle Modelle setzen auch stets liquide Märkte für vermögensbesicherte Wertpapiere voraus. Eine kritische Überprüfung, inwieweit die eingesetzten Modelle in einer Marktsituation, in der niemand mehr den Wertpapieren vertraut, noch zuverlässige Ergebnisse berechnen können, fand oftmals nicht statt. Insgesamt zeigten die Modelle nur sehr geringe Robustheit. Darüber hinaus verwendeten nahezu alle Banken, Investoren und Ratingagenturen dieselben oder zumindest sehr ähnliche Modelle zur Bewertung und Steuerung der strukturierten Wertpapiere. Dementsprechend hatte ein Großteil der involvierten Marktteilnehmer sehr ähnliche Erwartungen an die Performance der vermögensbesicherten Wertpapiere. Dies führte schließlich zu einer stark steigenden Nachfrage dieser Finanztitel. Da jedoch nahezu alle Beteiligten den gleichen Modellen vertrauten, übersahen viele Banken und Investoren die tatsächlichen Risiken, die die Wertpapiere bei ungünstigen Marktentwicklungen in sich bergen. So konnte die Blase um vermögensbesicherte Wertpapiere entstehen und letztendlich auch platzen.

Was können wir aus dem Beispiel der Immobilienmarktkrise für die IT-basierte Entscheidungsunterstützung lernen? Es wird schnell klar, dass solche Krisen sowie die voran- und einhergehenden Fehlentscheidungen nicht durch die Initiative Einzelner vermieden werden können, sondern es eines koordinierten Zusammenwirkens von Wissenschaft, Wirtschaft und Politik bedarf.

- Aus einer wissenschaftlichen Perspektive gilt es, insbesondere modelltheoretische Arbeiten stets einer kritischen Prüfung bzgl. Aussagekraft, Limitationen, Anwendbarkeit etc. zu unterziehen. In Beiträgen müssen die Annahmen sorgfältig und explizit herausgearbeitet werden und auf deren Basis entsprechende Limitationen der Modelle sowie deren Robustheit im Rahmen einer kritischen Würdigung abgeleitet werden. In vielen Fällen geschieht dies bereits, jedoch zeigt die Krise an den Immobilienkreditmärkten mit ihren weltweiten Folgen, dass ein noch größeres Augenmerk auf diesen Aspekt gelegt werden muss.

- Die Praxis muss sich auf die Verantwortung ihrer Entscheidungsträger besinnen. Ein blindes Vertrauen auf EUS und die darin integrierten Modelle birgt die Gefahr schwerwiegender Fehlentscheidungen. So können innovative Modelle zur Entscheidungsunterstützung und entsprechende Anwendungssysteme für ein erfolgreiches unternehmerisches Handeln zwar notwendig, aber niemals hinreichend sein. Die Verantwortung muss bei den Entscheidungsträgern liegen und dies erfordert einen differenzierten und reflektierten Umgang mit den entsprechenden Modellen, EUS und den generierten Handlungsempfehlungen.

- Aus politischer Sicht besteht einerseits die Herausforderung, die Folgen solcher Krisen soweit wie möglich und sinnvoll einzudämmen sowie andererseits das Auftreten neuer Krisen zu verhindern. Auch wenn Regulierung und Aufsichtsgremien in großem Maße einer blinden Modellgläubigkeit entgegenwirken können, können diese alleine sie nicht verhindern. Daher muss sowohl in der Wissenschaft als 
auch in der Praxis das Bewusstsein für einen entsprechenden reflektierten Umgang und eine differenzierte Bewertung von Modellen und durch Anwendungssysteme generierte Handlungsempfehlungen geschaffen werden. Dies kann nur durch eine Förderung entsprechender Forschungsaktivitäten und eine noch engere Verzahnung von Forschung und Praxis gelingen. Laut Nobelpreisträger Joseph Stiglitz wäre bspw. die Gründung einer Behörde wünschenswert, die - ähnlich wie bei Medikamenten - in enger Zusammenarbeit mit der Wissenschaft neuartige Finanzprodukte sowie die dahinter liegenden Modelle prüft und testet, bevor diese am Markt zugelassen werden.

Welchen konkreten Beitrag kann und muss die Wirtschaftsinformatik leisten? Wie weit geht unsere Verantwortung?

Als Interdisziplin zwischen BWL und Informatik kommt der Wirtschaftsinformatik bei der Erforschung und Gestaltung von Anwendungs- und Entscheidungsunterstützungssystemen sowie bei der Integration teilweise fachfremder Modelle eine besondere Verantwortung zu. Das mögliche Argument, in obigem Beispiel seien die ursächlichen Modelle rein finanzmathematischer Natur und damit nicht originär der Wirtschaftsinformatik zuzuordnen, greift zu kurz. Denn es sind die von Wirtschaftsinformatikern konzipierten EUS, die auf Basis dieser Modelle Informationen selektieren und Handlungsempfehlungen generieren. Da diese Anwendungssysteme als Teil von IKS den primären Gegenstand unserer Disziplin bilden, obliegt uns zumindest auch eine Mitverantwortung für deren fachliche Anwendungslogik. Darauf aufbauend müssen wir EUS derart gestalten, dass die Versuchung blinder Modellgläubigkeit weitestgehend reduziert wird.

Im Sinne des Wissenschaft-Praxis-Transfers zeichnet die Wirtschaftsinformatik ebenfalls für die Evaluation von Anwendungssystemen bzw. EUS und den darin umgesetzten Modellen unter Realweltbedingungen verantwortlich. Wir müssen kritisches Feedback bzgl. der praktischen Anwendbarkeit von Modellen und deren Robustheit unter Berücksichtigung der Modellannahmen an die "Ursprungsdisziplinen“ und die bereits vorhandenen Anwender zurückspielen. Nur so ist eine fruchtbare und evolutionäre Verknüpfung zum beiderseitigen Nutzen von Wissenschaft und Praxis im Sinne eines geschlossenen Kreislaufs möglich. Auch wenn uns dafür im Einzelfall die Detailkenntnisse fehlen, sollten wir unseren natürlichen Verstand einsetzen, denn nach Arthur Schopenhauer gilt:„Natürlicher Verstand kann fast jeden Grad von Bildung ersetzen, aber keine Bildung den natürlichen Verstand."

Nur mit diesem Anspruch können wir unsere - vielleicht nicht auf den ersten Blick ersichtliche, aber dennoch immanente - Verantwortung wahrnehmen und einen Beitrag zur Vermeidung zukünftiger Krisen leisten. Der interdisziplinären Ausrichtung der Wirtschaftsinformatik wohnt nicht nur eine Stärke inne, sondern auch eine entsprechende Verantwortung.

Prof. Dr. Hans Ulrich Buhl 